\title{
Association between body mass index and chronic kidney disease: A population-based, cross-sectional study of a Japanese community
}

\author{
Ikuo Nomura' \\ Johji Kato \\ Kazuo Kitamura' \\ 'Department of Internal Medicine, \\ Circulatory and Body Fluid \\ Regulation, Faculty of Medicine; \\ ${ }^{2}$ Frontier Science Research Center, \\ University of Miyazaki, Kiyotake, \\ Miyazaki, Japan
}

\begin{abstract}
Chronic kidney disease (CKD) has recently been recognized as a risk factor for cardiovascular disease, while the mechanism by which CKD develops remained to be clarified. In the present study, we conducted a cross-sectional, community-based study to identify the factor(s) associated with CKD. We examined 1978 local residents of the Kiyotake area of Japan (697 males and 1281 females; age, $60.8 \pm 11.0$ years; mean \pm SD), who had an annual health check-up. Diagnosis of CKD was made based on dipstick proteinuria of +1 or higher, or on a reduced glomerular filtration rate (GFR) estimated from serum creatinine level to less than 60 $\mathrm{ml} / \mathrm{min} / 1.73 \mathrm{~m}^{2}$. Body mass index (BMI) and prevalence of obesity in the residents with CKD were found to be higher than in those without CKD in both genders. When compared with the residents without $\mathrm{CKD}$, the ratios of residents taking antihypertensive medicines were higher in both genders, and the blood pressure and fasting blood glucose levels of males and the triglyceride level of females were elevated in those with CKD. These parameters or factors were found to be significant for CKD by a univariate logistic analysis. We further analyzed the data with a multivariate logistic method using age, BMI, antihypertensive and antidyslipidemic medicines, blood pressure, serum lipid and glucose as independent covariates, and found that BMI was a significant parameter independently correlated with CKD in both genders. Thus, increased BMI is associated with CKD independently of blood pressure, serum lipid and glucose levels in the general population.
\end{abstract}

Keywords: chronic kidney disease, body mass index, general population

\section{Introduction}

Chronic kidney disease (CKD) is defined as either structural and/or functional abnormality of the kidney or reduced glomerular filtration rate (GFR) to a level less than $60 \mathrm{ml} / \mathrm{min} / 1.73 \mathrm{~m}^{2}{ }^{1,2} \mathrm{CKD}$ has recently been recognized as a challenging public health issue not only because numbers of patients with end-stage renal disease (ESRD) are steadily increasing in developed countries including Japan, but also because recent epidemiological studies showed that CKD is a significant risk factor for cardiovascular disease. ${ }^{3-5}$ For example, the number of ESRD patients in Japan is currently greater than 250,000 , and this has become an economic burden for the health care system. ${ }^{6}$ On the other hand, it was shown in a Japanese community by a prospective study that ischemic heart disease and cerebral infarction are more frequent in the male and female residents with CKD, respectively, than in those without it. ${ }^{5}$ In order to prevent or reduce development of CKD at a community level, it is essential to identify or specify the cause(s) of CKD. Because GFR is reduced by aging, CKD is more frequent in elderly people than in younger ones. Besides aging, possible factors involved in the development of CKD are hypertension, impaired glucose tolerance or diabetes mellitus, dyslipidemia and obesity, ${ }^{7-14}$ but the detailed mechanism(s) remains to be clarified. In the present
Correspondence: Johji Kato Frontier Science Research Center, University of Miyazaki, 5200 Kihara, Kiyotake, Miyazaki 889-1692, Japan Tel +81985859718

Fax +8I 985856596

Email jkjpn@med.miyazaki-u.ac.jp 
study, we carried out a community-based, cross-sectional study of the local residents of the Kiyotake area in an attempt to identify the factor(s) associated with CKD.

\section{Materials and methods \\ Study subjects and protocol}

In this study, we examined local residents in the Kiyotake area, Miyazaki, Japan, who had an annual health check-up from September to October of 2006 responding to the recommendation made by the municipal government for those of 40 years or older. Upon visiting the community center of Kiyotake town, medical history of the subjects was taken by nurses and confirmed by physicians, and blood pressure was measured with an oscillometric automatic device (BP-103iII; Colin Japan Ltd, Tokyo, Japan) in a sitting position by experienced nurses. Urine was collected and blood was drawn from an antecubital vein after overnight fast. Serum lipid and glucose levels were measured by the automatic analyzer (Olympus AU2700; Olympus, Tokyo, Japan) with serum creatinine levels determined by the enzymatic method. GFRs were calculated with the formula of $0.741 \times 175 \times \mathrm{Age}^{-0.203} \times$ serum creatinine $\mathrm{s}^{-1.154}$ (further multiplied by 0.742 for females), the Modification of Diet in Renal Disease (MDRD) Study equation further modified for Japanese people by the Japanese Society of Nephrology. ${ }^{15,16}$ Residents were judged to have CKD when estimated GFR (eGFR) $<60 \mathrm{ml} / \mathrm{min} / 1.73 \mathrm{~m}^{2}$ or spot urine protein determined by dipstick measurement $\geq+1(30 \mathrm{mg} / \mathrm{dl})$. Obesity was defined with body mass index (BMI) of $25 \mathrm{~kg} / \mathrm{m}^{2}$ or larger, according to the criteria of the Japan Society for the Study of Obesity. ${ }^{17}$

In this health check-up program, 2085 residents (761 males, $63.1 \pm 10.7$ years; 1324 females, $59.8 \pm 10.9$ years; mean \pm SD) visited during the time period mentioned above. From those subjects, not to analyze CKD closely related with diabetic nephropathy, we excluded residents with fasting blood glucose levels of $126 \mathrm{mg} / \mathrm{dl}$ or higher $(\mathrm{n}=80)$, or those who were taking glucose-lowering agents or had been diagnosed as having diabetes mellitus by any physician $(\mathrm{n}=55)$. In addition, two male residents who had renal failure with eGFR less than $15 \mathrm{ml} / \mathrm{min} / 1.73 \mathrm{~m}^{2}$ were excluded from the analysis. As a result, we finally analyzed the data from 1978 local residents (697 males, $63.0 \pm 10.8$ years; 1281 females, $59.6 \pm 10.9$ years). This study was approved by the Review Committee of Cooperative and Commissioned Research and the Ethics Committee of University of Miyazaki Faculty of Medicine.

\section{Statistical analysis}

All the data were analyzed with SPSS software version 17.0 (SPSS Inc., Chicago, IL). Comparisons of two groups were assessed by unpaired $t$-test or Chi-squared test, and the relationship between BMI and eGFR was evaluated by linear regression analysis. The data were further examined by univariate and then multivariate logistic methods to extract significant, independent factors for CKD. All the data were expressed as means $\pm \mathrm{SD}$ and a $\mathrm{P}$ value less than 0.05 was considered to be significant.

\section{Results}

Table 1 shows the basal profiles of the male and female residents examined in this study. As shown, 22.8\% of male and $15.7 \%$ of female residents were found to have $\mathrm{CKD}$, diagnosis of which was made by estimated GFR $<60 \mathrm{ml} / \mathrm{min} / 1.73 \mathrm{~m}^{2}$ in 330 residents, by dipstick proteinuria $\geq+1$ in 16 or by both in 14. Expectedly, the mean ages in the male and female residents with $C K D$ were higher $(\mathrm{P}<0.01)$ than in those without $\mathrm{CKD}$. When compared with the residents without CKD, BMI of both genders was slightly, but significantly higher in those with CKD, resulting in significantly increased prevalence of obesity. When analyzed by linear regression analysis, BMI was found to be significantly correlated with eGFR in both genders (Figure 1). Current smoker ratio in the male CKD residents was lower than in the controls $(\mathrm{P}<0.05)$, while no difference was noted in the female residents. Both the systolic and diastolic blood pressure levels in the CKD subjects were higher than in the controls in male $(\mathrm{P}<0.01)$, but this was not the case for female. Meanwhile, ratios of subjects who were taking antihypertensive medicines in the $\mathrm{CKD}$ residents were significantly higher than in those without $\mathrm{CKD}$ in both genders. The fasting blood glucose (FBG) levels of the males $(\mathrm{P}<0.05)$ and the triglyceride level of the females $(\mathrm{P}<0.01)$ with $\mathrm{CKD}$ were found to be higher, compared with those without it, while there were no significant differences in ratio of the residents taking antidyslipidemic medicines. The number of residents who had a family history of cardiovascular diseases (CVD) was slightly higher in the residents with CKD in both genders, but the differences were not statistically significant.

The results of univariate and multivariate logistic analyses are shown in Table 2 for males and in Table 3 for females. In the univariate analyses, age, BMI and antihypertensive medication were found to be significant for CKD in both genders, and in addition, judged to be significant were smoking, blood pressure and FBG for males and triglyceride for females. Because differences were noted in smoking, blood pressure, antihypertensive medication, triglyceride, and FBG between those with and without CKD, we next analyzed the data by means of a multivariate logistic analysis using CKD as a dependent variable and the parameters listed 
Table I Basal profiles of male and female residents with or without CKD

\begin{tabular}{|c|c|c|c|c|}
\hline \multirow[t]{2}{*}{ CKD } & \multicolumn{2}{|l|}{ Male } & \multicolumn{2}{|l|}{ Female } \\
\hline & $(-)$ & $(+)$ & $(-)$ & $(+)$ \\
\hline $\mathrm{n}$ & 538 & 159 & 1080 & 201 \\
\hline Age (year) & $61.8 \pm 10.8$ & $66.9 \pm 9.8^{b}$ & $58.7 \pm 10.4$ & $64.7 \pm 12.1^{b}$ \\
\hline BMI $\left(\mathrm{Kg} / \mathrm{m}^{2}\right)$ & $20.0 \pm 2.8$ & $23.9 \pm 2.8^{b}$ & $21.9 \pm 3.1$ & $22.5 \pm 3.2^{\mathrm{a}}$ \\
\hline Obesity (\%) & 23.2 & $32.1^{\mathrm{a}}$ & 13.5 & $18.9^{\mathrm{a}}$ \\
\hline Smoking (\%) & 28.3 & $18.9^{a}$ & 3.1 & 2.0 \\
\hline $\mathrm{SBP}(\mathrm{mmHg})$ & $128 \pm 18$ & $135 \pm 18^{b}$ & $122 \pm 18$ & $125 \pm 17$ \\
\hline $\mathrm{DBP}(\mathrm{mmHg})$ & $79 \pm 10$ & $82 \pm 10^{b}$ & $73 \pm 10$ & $73 \pm 9$ \\
\hline Antihypertensive medicine (\%) & 17.3 & $27.0^{\mathrm{b}}$ & 12.1 & $21.4^{b}$ \\
\hline Total cholesterol (mg/dl) & $199 \pm 32$ & $201 \pm 31$ & $214 \pm 35$ & $215 \pm 36$ \\
\hline HDL-cholesterol (mg/dl) & $56 \pm 13$ & $54 \pm 12$ & $64 \pm 14$ & $62 \pm 16$ \\
\hline Triglyceride (mg/dl) & $112 \pm 80$ & $123 \pm 83$ & $91 \pm 45$ & $10 \mid \pm 56^{\mathrm{a}}$ \\
\hline Antidyslipidemic medicine (\%) & 2.2 & 2.5 & 7.5 & 7.5 \\
\hline FBG (mg/dl) & $94.5 \pm 9.1$ & $96.5 \pm 9.8^{\mathrm{a}}$ & $91.4 \pm 8.5$ & $91.7 \pm 7.9$ \\
\hline $\operatorname{EGFR}\left(\mathrm{ml} / \mathrm{min} / 1.73 \mathrm{~m}^{2}\right)$ & $72 \pm 10$ & $54 \pm 8^{b}$ & $75 \pm 12$ & $53 \pm 8^{b}$ \\
\hline Family history of CVD (\%) & 20.3 & 27.7 & 23.4 & 28.4 \\
\hline
\end{tabular}

Abbreviations: BMI, body mass index; SBP and DBP, systolic and diastolic blood pressure; HDL, high-density lipoprotein; FBG, fasting blood glucose; eGFR, estimated GFR; CVD, cardiovascular disease.

Definition of obesity, BMI $>=25.0 \mathrm{Kg} / \mathrm{m}^{2}$; Mean+l-SD; ${ }^{\text {P }}<0.05$, ${ }^{\text {b }}<0.01$, vs male or female residents without CKD.

in Tables 2 and 3 as independent covariates. As shown, smoking, blood pressure, antihypertensive medication, and FBG became insignificant, but BMI was found to be independently significant for $\mathrm{CKD}$ as well as age in both genders, and in females, triglyceride was a significant factor.

\section{Discussion}

Chronic kidney disease (CKD) has recently been recognized not only as a risk factor of ESRD, but also of CVD which is a leading cause of death in developed countries. ${ }^{1,2,6} \mathrm{An}$ interventional approach is necessary to prevent development of CKD at a community level, while the detailed mechanisms by which CKD develops remained to be specified. In the present study, we conducted this cross-sectional, observational study in the Kiyotake area, a Japanese community, and found that an increase in BMI is a significant factor independently associated with CKD.

Age is an important factor closely related with CKD because GFR is reduced by aging, while a number of factors have been shown to be associated with CKD besides aging. These are hypertension, impaired glucose tolerance or diabetes mellitus, dyslipidemia, obesity, and smoking. ${ }^{714}$ In the present study, to avoid including CKD secondary to diabetic nephropathy, we excluded subjects with diabetes mellitus or with a fasting blood glucose level of $126 \mathrm{mg} / \mathrm{dl}$ or higher. As shown in the basal profiles of the residents with CKD, the blood pressure and FBG level of males and the triglyceride level of females were significantly elevated, and the ratios of subjects taking antihypertensive medicine were higher in both genders, compared with those without CKD. Indeed, these parameters were significant for CKD in the univariate logistic analysis. But in the multivariate analysis, they were judged to be insignificant except for triglyceride in females, while BMI, as well as age, was extracted as an independently significant factor for CKD.

Hypertension has been clearly shown to be an important risk factor for future reduction in GFR and development of $\mathrm{ESRD},{ }^{7,8}$ but we found no significant association between CKD and blood pressure in the present study despite the fact that both the systolic and diastolic blood pressure levels were higher in the males with CKD than without CKD. The lack of association can be attributed to the close relationship between age and blood pressure levels, because blood pressure was judged to be significant for CKD by univariate analysis, but became insignificant when adjusted with the other parameters including age (Table 2). Obesity has also been recognized as a factor related with $\mathrm{CKD}$, but there has been no clear answer to an important question: what is the underlying mechanism of CKD developing in association with increased BMI. Assumed to be involved are the activated renin-angiotensin system, increased sympathetic nerve activity, insulin-resistance or hyperinsulinemia and dyslipidemia. ${ }^{7-14,18}$ It was reported that impaired glucose tolerance (IGT) is associated with development of CKD. ${ }^{11,19}$ 


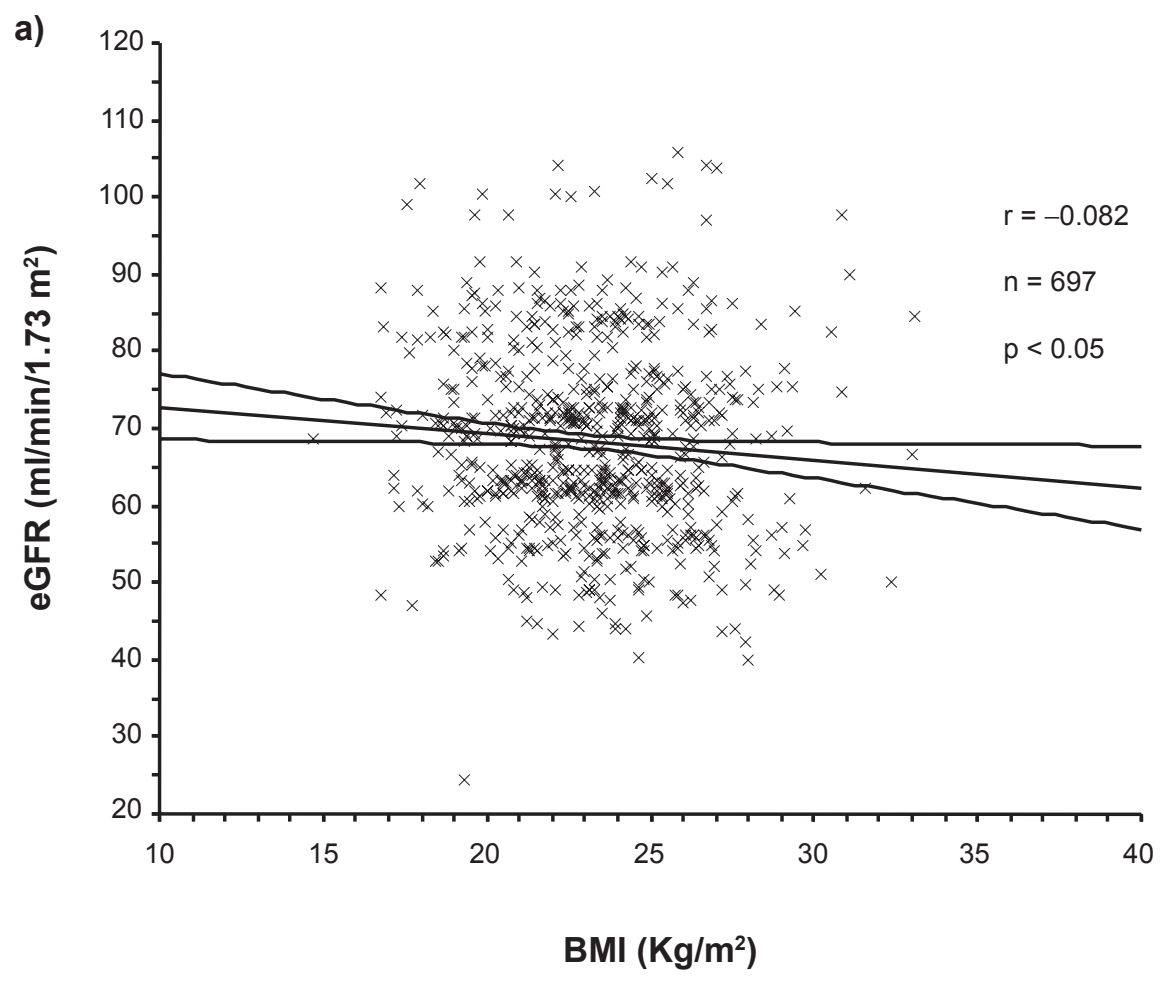

b)

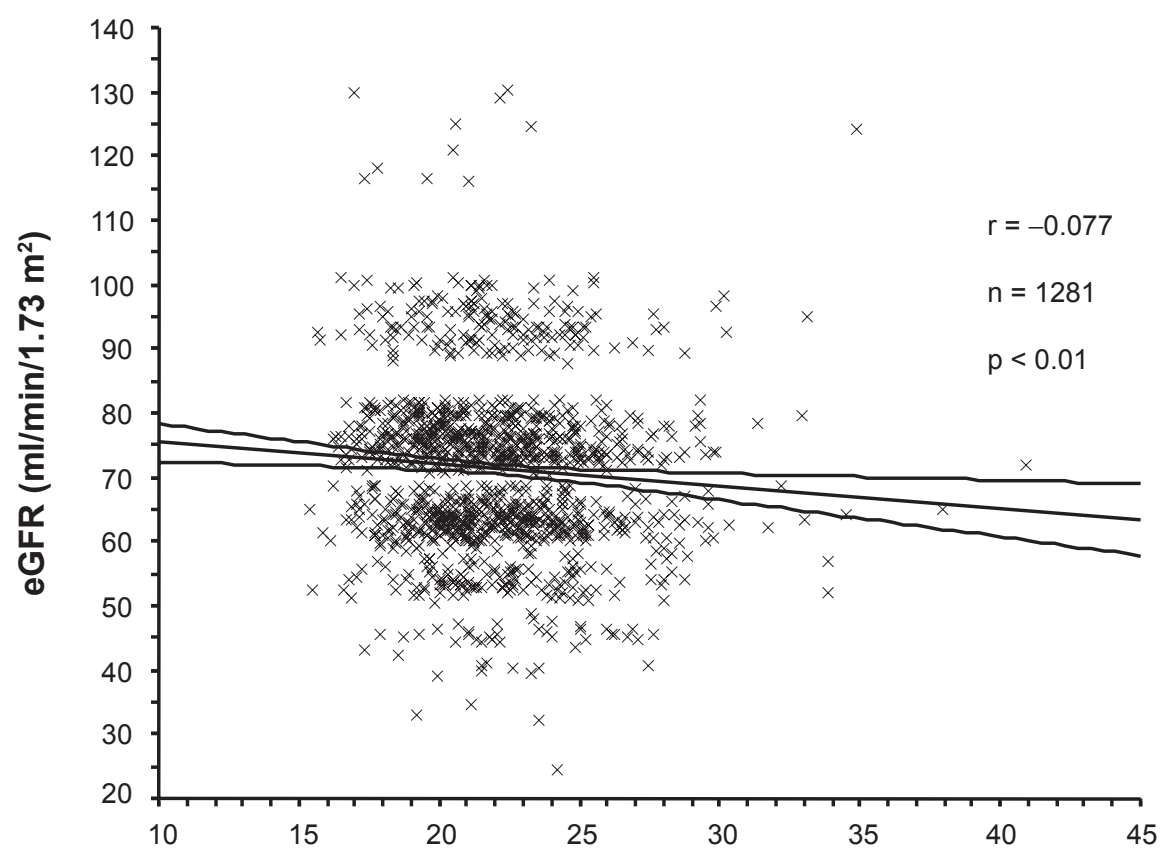

BMI $\left(\mathrm{Kg} / \mathrm{m}^{2}\right)$

Figure I Linear regression analyses of relationship between eGFR and BMI in the male a) and female b) residents. Regression lines and $95 \%$ confidence limits are shown in each graph.

Abbreviations: BMI, body mass index; eGFR, estimated glomerular filtration rate. 
Table 2 Identification of factor(s) associated with CKD by univariate and multivariate logistic analyses in male subjects

\begin{tabular}{|c|c|c|c|c|}
\hline \multirow{2}{*}{$\begin{array}{l}\text { Male } \\
\text { Parameters }\end{array}$} & \multicolumn{2}{|l|}{ Univariate } & \multicolumn{2}{|c|}{ Multivariate } \\
\hline & Odds ratio & $95 \% \mathrm{Cl}$ & Odds ratio & $95 \% \mathrm{Cl}$ \\
\hline Age (year) & $1.048^{\mathrm{b}}$ & $1.029-1.067$ & $1.062^{b}$ & $1.038-1.088$ \\
\hline BMI $\left(\mathrm{Kg} / \mathrm{m}^{2}\right)$ & $1.125^{\mathrm{b}}$ & $1.055-1.200$ & $1.139^{b}$ & $1.055-1.230$ \\
\hline Smoking ${ }^{c}$ & $0.591^{a}$ & $0.38 I-0.916$ & 0.801 & $0.500-1.282$ \\
\hline $\mathrm{SBP}(\mathrm{mmHg})$ & $1.020^{\mathrm{b}}$ & $1.010-1.030$ & 0.996 & $0.979-1.013$ \\
\hline $\mathrm{DBP}(\mathrm{mmHg})$ & $1.027^{\mathrm{b}}$ & $1.009-1.046$ & 1.021 & $0.992-1.05 \mid$ \\
\hline Antihypertensive medicine $^{c}$ & $1.774^{\mathrm{b}}$ & I. $|7|-2.687$ & 1.108 & $0.695-1.766$ \\
\hline Total cholesterol (mg/dl) & 1.002 & $0.997-1.008$ & 1.003 & $0.997-1.010$ \\
\hline HDL-cholesterol (mg/dl) & 0.988 & $0.974-1.001$ & 0.996 & $0.979-1.013$ \\
\hline Triglyceride (mg/dl) & 1.001 & $0.999-1.004$ & 1.002 & $0.999-1.004$ \\
\hline Antidyslipidemic medicine ${ }^{c}$ & 1.131 & $0.360-3.557$ & 0.698 & $0.209-2.325$ \\
\hline FBG (mg/dl) & $1.022^{\mathrm{a}}$ & $|.003-1.04|$ & $\mathrm{I} .00 \mathrm{I}$ & $0.981-1.022$ \\
\hline
\end{tabular}

Abbreviations: BMI, body mass index; SBP and DBP, systolic and diastolic blood pressure; HDL, high-density lipoprotein; FBG, fasting blood glucose.

Notes: ${ }^{\mathrm{P}}<0.05,{ }^{\mathrm{b}} \mathrm{P}<0.0 \mathrm{I}$, ${ }^{\mathrm{C} S m o k i n g}$, ${ }^{\mathrm{C}}$ Antihypertensive medicine, ${ }^{\mathrm{C}}$ Antidyslipidemic medicine, ( $\mathrm{no}=\mathrm{I}$ and yes $=2$ ).

The residents with diabetes mellitus or with a fasting blood glucose level of $126 \mathrm{mg} / \mathrm{dl}$ or higher were excluded, while neither glucose tolerance test nor measurement of insulin levels was done in the present study. Therefore, either IGT or hyperinsulinemia associated with increased BMI may be a possible explanation for the correlation between CKD and BMI. The independent association between CKD and triglyceride level in the female residents of this study appears to accord with this notion.

The present findings are also comparable with the report by Tanaka and colleagues, who showed that metabolic syndrome is an independent, significant determinant of CKD in a hospital-based screening program in Japan. ${ }^{20}$ In the present study, the differences in BMI or the percentages of obese subjects are relatively small between those with and without CKD, though BMI was extracted as a significant factor independent of blood pressure, serum HDL, triglyceride and FBG levels, which are important factors in criteria of metabolic syndrome. It has been thought that Asians including Japanese are susceptible to increased body weight in terms of development of metabolic disorders including diabetes mellitus: for example, there is a notable difference between the definition of obesity for Caucasians and that for Japanese. ${ }^{17,21}$ It is possible that Japanese are also susceptible to increased body weight in terms of development of CKD, but this hypothesis, as well as the detailed mechanisms of development of CKD associated with increased BMI, should be clarified by future studies. Recently it was reported that

Table 3 Identification of factor(s) associated with CKD by univariate and multivariate logistic analyses in female subjects

\begin{tabular}{|c|c|c|c|c|}
\hline \multirow{2}{*}{$\begin{array}{l}\text { Female } \\
\text { Parameters }\end{array}$} & \multicolumn{2}{|l|}{ Univariate } & \multicolumn{2}{|c|}{ Multivariate } \\
\hline & Odds ratio & $95 \% \mathrm{Cl}$ & Odds ratio & $95 \% \mathrm{Cl}$ \\
\hline Age (year) & $1.054^{\mathrm{b}}$ & $1.038-1.069$ & $1.062^{b}$ & $1.043-1.081$ \\
\hline $\mathrm{BMI}\left(\mathrm{Kg} / \mathrm{m}^{2}\right)$ & $1.056^{\mathrm{a}}$ & I.008-I.107 & $\left.1.07\right|^{a}$ & $1.013-1.132$ \\
\hline Smoking ${ }^{c}$ & 0.625 & $0.219-1.780$ & 0.795 & $0.272-2.321$ \\
\hline $\mathrm{SBP}(\mathrm{mmHg})$ & 1.007 & $0.999-1.016$ & 0.991 & $0.976-1.006$ \\
\hline $\mathrm{DBP}(\mathrm{mmHg})$ & 1.001 & $0.986-1.016$ & 0.995 & $0.972-1.020$ \\
\hline Antihypertensive medicine ${ }^{c}$ & $1.972^{\mathrm{b}}$ & $1.344-2.893$ & 1.312 & $0.852-2.021$ \\
\hline Total cholesterol (mg/dl) & 1.002 & $0.997-1.006$ & 1.000 & $0.994-1.005$ \\
\hline HDL-cholesterol (mg/dl) & 0.993 & $0.982-1.003$ & 1.008 & $0.994-1.022$ \\
\hline Triglyceride (mg/dl) & $1.004^{b}$ & $1.001-1.007$ & $1.004^{\mathrm{a}}$ & $1.000-1.008$ \\
\hline Antidyslipidemic medicine ${ }^{c}$ & 0.994 & $0.560-1.762$ & 0.620 & $0.34 I-1.128$ \\
\hline FBG (mg/dl) & 1.004 & $0.986-1.022$ & 0.981 & $0.961-1.001$ \\
\hline
\end{tabular}

Abbreviations: BMI, body mass index; SBP and DBP, systolic and diastolic blood pressure; HDL, high-density lipoprotein; FBG, fasting blood glucose.

Notes: ${ }^{\mathrm{P}}<0.05,{ }^{\mathrm{b}} \mathrm{P}<0.01$; ' $\mathrm{C}$ moking, ${ }^{\mathrm{c} A n t i h y p e r t e n s i v e ~ m e d i c i n e, ~}{ }^{\mathrm{C}}$ Antidyslipidemic medicine ( $\mathrm{no}=\mathrm{I}$ and yes $=2$ ). 
significant association between CKD and BMI was found in men but not in women by two epidemiological studies done by Ishizaka and colleagues in Japan and by Shankar and colleagues in Singapore. ${ }^{22,23}$ The significant association found in the females of the present study is inconsistent with those reports. We have no clear explanation for this discrepancy, while may be able to raise a possibility of difference in age of study subjects. The mean age of the female subjects examined by Ishizaka and colleagues was younger than that of the present study. Age of female subjects may be an important factor influencing results of this type of epidemiological study, because estrogens, blood levels of which decline after menopause, have been shown to exert a protective action on kidneys. ${ }^{24}$

Lastly, we should mention the limitations of the present study. First, the subjects examined in the present study were local residents, who had the health check-up in response to the recommendation of the municipal government. Therefore, it should be noted that the subjects examined are not necessarily a group of people representative of those residing in the Kiyotake area in terms of basal profiles of cardiovascular risk factors, such as age and CKD. Next, this is a simply observational study examining the cross-sectional relationship between BMI and CKD, and interventional, follow-up studies are required to see the effect of body weight reduction on development of CKD.

\section{Acknowledgments}

We are grateful to Mr Kazutoshi Abumi, the Mayor of Kiyotake, and the staff of Kiyotake Town Department of Welfare and Health for their assistance. The authors report no conflicts of interest in this work.

\section{References}

1. National Kidney Foundation. K/DOQI clinical practice guidelines for chronic kidney disease: evaluation, classification, and stratification. Am J Kidney Dis. 2002;39(2 Suppl 1):S1-S266.

2. Levey AS, Coresh J, Balk E, et al. National Kidney Foundation practice guidelines for chronic kidney disease: evaluation, classification, and stratification. Ann Intern Med. 2003;139(2):137-147.

3. Sarnak MJ, Levey AS, Schoolwerth AC, et al. Kidney disease as a risk factor for development of cardiovascular disease: a statement from the American Heart Association Councils on Kidney in Cardiovascular Disease, High Blood Pressure Research, Clinical Cardiology, and Epidemiology and Prevention. Hypertension. 2003;42(5):1050-1065.

4. Go AS, Chertow GM, Fan D, McCulloch CE, Hsu CY. Chronic kidney disease and the risks of death, cardiovascular events, and hospitalization. N Engl J Med. 2004;351(13):1296-1305.
5. Ninomiya T, Kiyohara Y, Kubo M, et al. Chronic kidney disease and cardiovascular disease in a general Japanese population: the Hisayama Study. Kidney Int. 2005;68(1):228-236.

6. Imai E, Horio M, Iseki K, et al. Prevalence of chronic kidney disease (CKD) in the Japanese general population predicted by the MDRD equation modified by a Japanese coefficient. Clin Exp Nephrol. 2007;11(2):156-163.

7. Klag MJ, Whelton PK, Randall BL, et al. Blood pressure and end-stage renal disease in men. $N$ Engl J Med. 1996;334(1):13-18.

8. Iseki K, Iseki C, Ikemiya Y, Kinjo K, Takishita S. Risk of developing low glomerular filtration rate or elevated serum creatinine in a screened cohort in Okinawa, Japan. Hypertens Res. 2007;30(2):167-174.

9. Chen J, Muntner P, Hamm LL, Jones DW, Batuman V, Fonseca V. The metabolic syndrome and chronic kidney disease in US adults. Ann Intern Med. 2004;140(3):167-174.

10. Hallan S, de Mutsert R, Carlsen S, Dekker FW, Aasarød K, Holmen J. Obesity, smoking, and physical inactivity as risk factors for CKD: are men more vulnerable? Am J Kidney Dis. 2006;47(3):396-405.

11. Kubo M, Kiyohara Y, Kato I, et al. Effect of hyperinsulinemia on renal function in a general Japanese population: the Hisayama study. Kidney Int. 1999;55(6):2450-2456.

12. Nguyen $\mathrm{S}$, Hsu CY. Excess weight as a risk factor for kidney failure. Curr Opin Nephrol Hypertens. 2007;16(2):71-76.

13. Kramer H, Luke A, Bidani A, Cao G, Cooper R, McGee D. Obesity and prevalent and incident CKD: the Hypertension Detection and Follow-Up Program. Am J Kidney Dis. 2005;46(4):587-594.

14. Yamagata K, Ishida $K$, Sairenchi T, et al. Risk factors for chronic kidney disease in a community-based population: a 10-year follow-up study. Kidney Int. 2007;71(2):159-166.

15. Levey AS, Coresh J, Greene T, et al; for the Chronic Kidney Disease Epidemiology Collaboration. Using standardized serum creatinine values in the modification of diet in renal disease study equation for estimating glomerular filtration rate. Ann Intern Med. 2006;145(4): 247-254.

16. Imai E, Horio M, Nitta K, et al. Estimation of glomerular filtration rate by the MDRD study equation modified for Japanese patients with chronic kidney disease. Clin Exp Nephrol. 2007;11(1):41-50.

17. Examination Committee of Criteria for 'Obesity Disease' in Japan, Japan Society for the Study of Obesity: New criteria for 'obesity disease' in Japan. Circ J. 2002;66(11):987-992.

18. Kambham N, Markowitz GS, Valeri AM, Lin J, D’Agati VD. Obesityrelated glomerulopathy: an emerging epidemic. Kidney Int. 2001;59(4): $1498-1509$.

19. Fox CS, Larson MG, Leip EP, Meigs JB, Wilson PWF, Levy D. Glycemic status and development of kidney disease. The Framingham Heart Study. Diabetes Care. 2005;28(10):2436-2440.

20. Tanaka H, Shiohira Y, Uezu Y, Higa A, Iseki K. Metabolic syndrome and chronic kidney disease in Okinawa, Japan. Kidney Int. 2006;69(2):369-374.

21. Dhiman RK, Duseja A, Chawla Y. Asians need different criteria for defining overweight and obesity. Arch Intern Med. 2005;165(9):1069-1070.

22. Ishizaka N, Ishizaka $\mathrm{Y}$, Toda E, et al. Association between obesity and chronic kidney disease in Japanese: differences in gender and hypertensive status? Hypertens Res. 2007;30(11):1059-1064.

23. Shankar A, Leng C, Chia KS, et al. Association between body mass index and chronic kidney disease in men and women: populationbased study of Malay adults in Singapore. Nephrol Dial Transplant. 2008;23(6):1910-1918.

24. Neugarten J, Silbiger S, Golestaneh L. Gender and kidney disease. In: Brenner BM, editor. The Kidney. Philadelphia, PA: Saunders; 2008. p. $674-680$. 